VOL. II (1974), 395-4II.

\title{
Multifunctions of Souslin type
}

\section{S.J. Leese}

Let $S$ and $X$ be any two sets; then a mapping. $\Gamma$ which assigns to each point $t$ in $S$ a set $\Gamma(t)$ of points in $X$ is called a multifunction from $S$ into $X$. A selector for $\Gamma$ is a function $f$ from $S$ into $X$ such that $f(t) \in \Gamma(t)$ for each $t$. We introduce here a class of multifunctions which is both well-supplied with measurable selectors and yet is comprehensive enough to include those kinds of multifunction which have been most commonly studied before. Hence in order to show that a multifunction with non-empty values, which may arise naturally in an implicit function problem, has a measurable selector, it is sufficient to show that it is of Souslin type.

\section{Introduction}

A problem which has often arisen in control theory (for example, [5], [10]) and mathematical economics (for example, [1], [4]) is the following. given a measurable space $S$ and a topological space $X$, what is a sufficient condition for a subset $A$ of $S \times X$ to have a measurable selector (that is, a measurable function $f: S \rightarrow X$ such that $(t, f(t)) \in A$ for all $t \in S)$ ?

We introduce here the class of multifunctions of Souslin type and show (Theorem 7) that if the set $A$ is the graph of such a multifunction and if the projection of $A$ on $S$ is all of $S$, then there is a measurable selector. This class of multifunctions is shown to include those which are

Received $31 \mathrm{July} 1974$. This work was done while the author was registered for the degree of $\mathrm{PhD}$ at the University of Keele and enjoying the research facilities of the University of Western Australia. The author is grateful to the Science Research Council for financial support, and also to Professor A.P. Robertson for his helpful suggestions and encouragement. 
studied in [3], [7], [12], and [14], and so the various selection theorems and implicit function theorems of those papers (and also of [1] and [11]) follow from our Theorem 7. The class of multifunctions of Souslin type is stable with respect to the usual operations of analysis; its name was given because of the parallels between this theory and the classical theory of Souslin sets ([2], [8]).

\section{Definitions and notation}

In what follows $S$ will be a measurable space; that is, a set on which is defined a $\sigma$-algebra $M$ of subsets which we shall call measurable. $X$ will denote a topological space and $B_{X}$ the $\sigma$-algebra generated by the closed sets of $X$; the sets in $B_{X}$ are called the Borel sets of $X$. A multifunction $\Gamma$ from $S$ into $X$ (which we shall abbreviate to $\Gamma: S \rightarrow X$ ) is a mapping from $S$ into the space of subsets of $X$. The graph of $\Gamma$ is the set

$$
G(\Gamma)=\{(t, x) \in S \times X: x \in \Gamma(t)\} .
$$

To avoid confusion with point-valued functions, we shall denote multifunctions by upper-case Greek letters and mappings by lower-case Greek or Roman letters. The multifunction $\Gamma$ will be said to be measurable ([3], [7], [12]) if for every closed set $F$ in $X$ the set

$$
\Gamma^{-}(F)=\{t \in S: \Gamma(t) \cap F \neq \emptyset\}
$$

is measurable. This is equivalent to the condition that for every open set $G$ in $X$ the set

$$
\Gamma^{+}(G)=\{t \in S: \Gamma(t) \subset G\}
$$

is measurable. We shall make use of the Souslin operation, which is fully discussed in [6] and [8]: iet $\left(A_{\sigma_{1} \ldots \sigma_{n}}\right)$ be a countable collection of sets in a given space, indexed by the set of all finite sequences $\sigma_{1}, \ldots, \sigma_{n}$ of positive integers. Then the set

$$
A=\bigcup_{\sigma} \bigcap_{n=1}^{\infty} A_{\sigma_{1}} \ldots \sigma_{n},
$$

the union being taken over the collection of all infinite sequences $\sigma$ of 
positive integers, is said to be obtained from the collection $\left(A_{\sigma_{1}} \ldots \sigma_{n}\right)$ by the Souslin operation. If the sets $\left(A_{\sigma_{1}} \ldots \sigma_{n}\right)$ belong to a given class $N$ of sets, then $A$ will be said to belong to the class Souslin-N . We shall use the notation of [13], pp. 44-49, and write

$$
A=\mathrm{U}_{\sigma}^{\mathrm{U}} \bigcap_{n=1}^{\infty} A_{\sigma \mid n},
$$

where $\sigma \mid n$ denotes the finite sequence $\sigma_{1}, \ldots, \sigma_{n}$.

The measurable space $S$ will be said to admit the Souslin operation if every subset formed in this way from measurable sets is measurable. Every measurable space derived from an outer measure admits the Souslin operation; this is proved in [13], pp. 44-49. Moreover, it follows from the remarks on p. 95 of [8] that if $S$ is a locally compact Hausdorf $f$ space with a Radon measure $\mu$, then the class of $\mu$-measurable sets admits the Souslin operation.

\section{Multifunctions of Souslin type}

If a topological space $P$ is separable and can be metrised so that it becomes a complete metric space, then $P$ is said to be a Polish space.

DEFINITION. Let $S$ be a measurable space, and $X$ a topological space. Then a multifunction $\Gamma: S \rightarrow X$ is said to be of Souslin type if there exists a Polish space $P$, a measurable multifunction $\Omega: S \rightarrow P$ with closed values, and a continuous mapping $\varphi: P \rightarrow X$ such that, for all $t, \Gamma(t)=\varphi(\Omega(t))$.

A multifunction of Souslin type is necessarily measurable.

EXAMPLES. (i) If $\Gamma: S \rightarrow X$ is a measurable closed-valued multifunction, and $X$ is a Polish space, then $\Gamma$ is of Souslin type.

(ii) If $\Gamma: S \rightarrow X$ is a compact-valued measurable multifunction, $X$ being a separable metrisable space, and $i: X \rightarrow \hat{X}$ is the embedding of $X$ into its completion $\hat{x}$ with respect to some suitable metric, then the multifunction $t \rightarrow i(\Gamma(t))$ is of Souslin type.

(iii) If $S$ admits the Souslin operation and $X$ is a regular 
continuous image of a Polish space $P$, then any measurable closed-valued multifunction $\Gamma: S \rightarrow X$ is of Souslin type. This follows from the fact that if $X=\varphi(P), \varphi$ being a continuous mapping, then the correspondence $t \rightarrow \varphi^{-1}(\Gamma(t))$ is also a measurable multifunction, as is proved in Lenma 1 of [12].

(iv) Let $(S, M)$ be a measurable space which admits the Souslin operation, and $X$ the continuous image of a Polish space; then if $\Gamma: S \rightarrow X$ is such that its graph belongs to the $\sigma$-algebra $M \otimes B_{X}$ generated by the sets $A \times B$, where $A$ is measurable and $B$ is Borel measurable, then $\Gamma$ is of Souslin type. This will be proved below (Lemma 3).

(v) The special class of multifunctions discussed in [7] is shown there, incidentally, to satisfy the conditions of our definition; thus they too are of Souslin type.

In what follows, $R$ will denote the class of all sets $A \times B$ where $A$ is a measurable set in $S$ and $B$ is a closed set in $X$. We shall need the following lenma, which is a generalization of result (3.4) of [4].

LEMMA 1. Let $X_{1}$ be a topological space and $K^{*}$ the class of sets which are closed and compact in $X_{1}$. Let $Y$ be a Souslin-K* subspace of $X_{1}$. Then if $S$ is a measurable space which admits the Souslin operation, and $\pi_{1}$ is the canonical projection from $S \times Y$ into $S, \pi_{1}(A)$ is measurable for every Souslin-R set $A$ in $S \times Y$.

Proof. Let

$$
A=\bigcup_{\sigma} \prod_{n=1}^{\infty}\left(A_{\sigma \mid n} \times B_{\sigma \mid n}\right),
$$

where each $A_{\sigma \mid n}$ is measurable and each $B_{\sigma \mid n}$ is closed relative to $Y$. Now for each $\sigma \mid n, B_{\sigma \mid n}=C_{\sigma \mid n} \cap Y$, say, where $C_{\sigma \mid n}$ is closed in $X$. Hence each $B_{\sigma \mid n}$ is Souslin-K* in $X_{1}$, and as the iteration of the Souslin operation produces no new sets $([6], \S 19), A$ is Souslin- $R$ in $S \times X$. Thus, with new notation, we may write 


$$
A=\bigcup_{\sigma} \bigcap_{n=1}^{\infty}\left(D_{\sigma \mid n} \times E_{\sigma \mid n}\right)
$$

where each $D_{\sigma \mid n}$ is measurable, and each $E_{\sigma \mid n}$ is a closed compact set in $x_{1}$. For each finite sequence $\sigma \mid n$, we define

$$
E_{\sigma \mid n}^{*}=E_{\sigma_{1}} \cap \ldots \cap E_{\sigma_{1}} \ldots \sigma_{n}
$$

and we put $D_{\sigma \mid n}^{*}=\emptyset$ if $E_{\sigma \mid n}^{*}=\varnothing, D_{\sigma \mid n}^{*}=D_{\sigma \mid n}$ otherwise. Then

$$
\begin{aligned}
A & =\underset{\sigma}{U} \bigcap_{n=1}^{\infty}\left(D_{\sigma \mid n}^{*} \times E_{\sigma \mid n}^{*}\right) \\
& \left.=U_{\sigma} \prod_{n=1}^{\infty} D_{\sigma \mid n}^{*}\right) \times\left(\begin{array}{cc}
\bigcap_{n=1}^{\infty} & E_{\sigma \mid n}^{*}
\end{array}\right) .
\end{aligned}
$$

Thus

$$
\begin{aligned}
\pi_{1}(A) & \left.=\underset{\sigma}{\cup} \pi_{1} \int_{n=1}^{\infty} D_{\sigma \mid n}^{*} \times \prod_{n=1}^{\infty} E_{\sigma \mid n}^{*}\right) \\
& =\underset{\sigma}{\cup} \prod_{n=1}^{\infty} D_{\sigma \mid n}^{*},
\end{aligned}
$$

since if $\prod_{n=1}^{\infty} E_{\sigma \mid n}^{*}$ is empty, $E_{\sigma \mid n}^{*}=\emptyset$ for some $n$, by compactness, in which case $D_{\sigma \mid n}^{*}=\varnothing$, by our definition. This completes the proof of the lemma.

COROLLARY 1. Let $X_{1}, S$, and $Y$ be as in Lemma 1. Then if $Z=\varphi(Y)$, where $\varphi$ is a continuous mapping, and $A$ is a Souslin-R set in $S \times Z, \pi_{1}(A)$ is measurable.

Proof. Let

$$
A=\bigcup_{\sigma} \prod_{n=1}^{\infty}\left(A_{\sigma \mid n} \times B_{\sigma \mid n}\right)
$$

where each $A_{\sigma \mid n}$ is measurable and each $B_{\sigma \mid n}$ is closed. We define 


$$
A_{1}=\underset{\sigma}{U} \prod_{n=1}^{\infty}\left(A_{\sigma \mid n^{x_{\varphi}}}{ }^{-1}\left(B_{\sigma \mid n}\right)\right)
$$

in $S \times Y$.

It is easily shown that $\pi_{1}(A)=\pi_{1}\left(A_{1}\right)$, whence the corollary 'follows immediately.

COROLLARY 2. If $S$ admits the Souslin operation, $P$ is a Polish space and $\Gamma: S \rightarrow P$ has Souslin-R graph, then $\Gamma$ is measurable.

Proof. If $F$ is a closed set in $P$,

$$
\Gamma^{-}(F)=\pi_{1}(G(\Gamma) \cap(S \times F)) \text {. }
$$

This is the projection of a Souslin-R set, and it follows from Lemma 1 that it is measurable, as by [2], p. 197, $P$ is homeomorphic to a countable intersection of open sets in the cube $I^{N}$, the product of the interval $[0,1]$ with itself countably many times.

This result is clearly still true if $P$ is the continuous image of a Polish space.

THEOREM 1. If $\left(\Gamma_{n}\right)$ is a sequence of multifunctions of Souslin type from any measurable space $S$ into the topological space $X$, then the multifunction $\Gamma: S \rightarrow X$ defined by

$$
\Gamma(t)=\bigcup_{n=1}^{\infty} \Gamma_{n}(t) \text { for each } t \text {, }
$$

is also of Souslin type.

Proof. Let the corresponding closed-valued multifunctions and mappings be

$$
\Omega_{n}: S \rightarrow P_{n}, \varphi_{n}: P_{n}+X, n=1,2, \ldots
$$

Let $P$ be the Polish space $\sum_{n=1}^{\infty} P_{n}$ ([2], p. 195) got by juxtaposing the spaces $P_{n}$ as disjoint closed subsets, and let $\varphi$ be the continuous mapping from $P$ to $X$ which coincides with $\varphi_{i}$ on $P_{i}$. 
Define $\Omega(t)=\sum_{n=1}^{\infty} \Omega_{n}(t)$ for each $t$ in $S$; then this is closedvalued and measurable, and moreover, $\varphi(\Omega(t))=\Gamma(t)$ for each $t$.

THEOREM 2. If $S$ is a measurable space which admits the Souslin operation, $\left(X_{i}\right)$ a sequence of topological spaces, and $\left(\Gamma_{i}\right)$ a sequence of multifunctions of Souslin type from $S$ into $x_{i}$ respectively; then the multifunction $\Gamma: S \rightarrow x_{1} \times x_{2} \times \ldots$ is also of Souslin type, where for each $t$,

$$
\Gamma(t)=\Gamma_{1}(t) \times \Gamma_{2}(t) \times \ldots .
$$

Proof. Let the corresponding closed-valued multifunctions and mappings be

$$
\Omega_{i}: S+P_{i}, \varphi_{i}: P_{i} \rightarrow X_{i}, i=1,2, \ldots .
$$

We then define

$$
\Omega(t)=\prod_{i=1}^{\infty} \Omega_{i}(t)
$$

and $P=P_{1} \times P_{2} \times \ldots$.

Then $P$ is a Polish space ([2], p. 195). Let $G=G_{1} \times G_{2} \times \ldots$ be any basic open set in $P$ (that is, $G_{i}=P_{i}$ for all but finitely many $i$. Each $G_{i}$ is open).

Then it is easily seen that

$$
\Omega^{-}(G)=\prod_{n=1}^{\infty} \Omega_{i}^{-}\left(G_{i}\right)
$$

which is a measurable set. Since any open set $B$ in $P$ is a countable union of basic ones $H_{i}(i=1,2, \ldots)$,

$$
\Omega^{-}(B)=\bigcup_{n=1}^{\infty} \Omega^{-}\left(B_{n}\right)
$$

which is measurable. Thus $\Omega$ is a measurable multifunction, by Theorem 3 
of [12]. We define a continuous mapping $\varphi$ from $P$ into $\Pi_{i} X_{i}$ by $\varphi\left(p_{1}, p_{2}, \ldots\right)=\left(\varphi_{1}\left(p_{1}\right), \varphi_{2}\left(p_{2}\right), \ldots\right),\left(p_{i} \in P_{i}\right)$.

Then $\Gamma(t)=\varphi(\Omega(t))$ for each $t$, and so $\Gamma$ is of Souslin type.

LEMMA 2. Let $X, Y$ be topological spaces, and $\psi: X \rightarrow Y$ a continuous mapping. Then if $S$ is a measurable space and $\Gamma: S \rightarrow X$ a multifinction of Souslin type, so is the multifunction $t \rightarrow \psi(\Gamma(t))$.

THEOREM 3. Let $S$ be a measurable space which admits the Souslin operation, $E$ a topological vector space, and $\Gamma_{1}, \Gamma_{2}$ multifunctions of Souslin type from $S$ into $E$. Then the multifunction $\Gamma: t \rightarrow \Gamma_{1}(t)+\Gamma_{2}(t)$ is of Souslin type. Moreover, if $\alpha$ is any measurable scalar-valued function on $S$, the multifunction $t \rightarrow \alpha(t) \Gamma_{1}(t)$ is also of Souslin type.

Proof. From Theorem 2, the multifunction $t \rightarrow \Gamma_{1}(t) \times \Gamma_{2}(t)$ is of Souslin type, from $S$ into $E \times E$. Now the mapping $\varphi: E \times E \rightarrow E$ defined by $\varphi(x, y)=x+y$ is continuous. Hence $\Gamma$ is of Souslin type, by Lenma 2, since, for each $t, \Gamma(t)=\varphi\left(\Gamma_{1}(t) \times \Gamma_{2}(t)\right)$.

To prove the second part of the theorem, we observe that by Theorem 2 , the multifunction $t \rightarrow\{\alpha(t)\} \times \Gamma_{1}(t)$ is of Souslin type. Now the mapping $\varphi:(\lambda, x) \rightarrow \lambda x$ is continuous, where $\lambda$ is in the field of scalars, and we have, for each $t$,

$$
\alpha(t) \Gamma_{1}(t)=\varphi\left(\{\alpha(t)\} \times \Gamma_{1}(t)\right),
$$

and hence the multifunction is of Souslin type, by Lenma 2 .

Clearly a could here be any multifunction of Souslin type into the field of scalars. Moreover, this theorem still holds if $E$ is any topological group and $\Gamma(t)=\Gamma_{1}(t) \cdot \Gamma_{2}(t)$ for each $t$.

THEOREM 4. Let $S$ be a measurable space which admits the Souslin operation, $E$ a topological vector space, and $\Gamma: S \rightarrow E$ a multifranction of Souslin type. Then the multifunction $\hat{\Gamma}$ where, for each $t, \hat{\Gamma}(t)$ is the convex hull of $\Gamma(t)$, is also of Souslin type. 
Proof. Let $\Lambda_{n}$ be the simplex in $R^{n}$ defined by:

$$
\lambda \in \Lambda_{n} \text { if and only if } \lambda_{i} \geq 0 \text { for all } i \text {, and } \sum_{i=1}^{n} \lambda_{i}=1 \text {. }
$$

We then define a sequence $\left(\Gamma_{n}\right)$ of multifunctions by taking, for each $n$ and each $t$,

$$
\Gamma_{n}(t)=\left\{\lambda_{1} x_{1}+\ldots+\lambda_{n} x_{n}: \lambda \in \Lambda_{n}, x_{i} \in \Gamma(t) \text {, for all } i\right\} .
$$

Let $\bar{E}^{n}$ denote the product of $E$ with itself, with $n$ factors. We define a mapping $\varphi_{n}: \Lambda_{n} \times E^{n} \rightarrow E$ by the formula:

$$
\varphi_{n}\left(\lambda ; x_{1}, \ldots, x_{n}\right)=\lambda_{1} x_{1}+\ldots+\lambda_{n} x_{n} .
$$

$\Phi_{n}$ is continuous, since $E$ is a topological vector space. By Theorem 2 the multifunction

$$
\Delta_{n}: t \rightarrow \Lambda_{n} \times \Gamma(t) \times \ldots \times \Gamma(t),
$$

where the factor $\Gamma(t)$ occurs $n$ times, is of Souslin type. Now for each $t, \Gamma_{n}(t)=\varphi_{n}\left(\Delta_{n}(t)\right)$, and so $\Gamma_{n}$ is of Souslin type, by Lemma 2 .

Therefore, since $\hat{\Gamma}(t)=\bigcup_{n=1}^{\infty} \Gamma_{n}(t)$, for each $t, \hat{\Gamma}$ is of Souslin type, by Theorem 1 .

COROLLARY. If, with the notation of Theorem 4, $E$ is metrisable, then the multifunction $\tilde{\Gamma}$, where, for each $t, \tilde{\Gamma}(t)$ is the closed convex hull of $\Gamma(t)$, is also of Souslin type.

Proof. For every open set $G$ in $E$,

$$
\hat{\Gamma}^{-}(G)=\tilde{\Gamma}^{-}(G) \text {, }
$$

and this is a measurable set, since $\hat{\Gamma}$ is measurable and $G$ is an $F_{\sigma}$. Hence $\tilde{\Gamma}$ is measurable, by Theorem 3 of [12]; and so is of Souslin type, by Example (iii).

These last two theorems extend the results on p. 107 of [3]. We now obtain a characterization of multifunctions of Souslin type by means of the 
Souslin operation.

THEOREM 5. Let $S$ be a measurable space, $X$ a Hausdorff topological space, and $\Gamma: S \rightarrow X$ a multifunction of Souslin type. Then the graph of $\Gamma$ is Souslin-R.

Proof. Let $P$ be a Polish space, and $d$ a metric for $P$ such that $(P, d)$ is a complete metric space. Let $\Omega: S \rightarrow X$ be a closed-valued measurable multifunction such that for each $t$,

$$
\Gamma(t)=\varphi(\Omega(t)) \text {. }
$$

We define a family $\left(U_{\sigma \mid n}\right)$ of closed sets of $P$ (called a sifting) as follows (this technique is also used in [12]): let $\left(U_{i}\right)$ be a countable covering of $P$ by closed sets of diameter $\leq 1 / 2$. Then for each $U_{i}$, let the collection $\left\{U_{i j}: j=1,2, \ldots\right\}$ be a covering of $U_{i}$ by closed sets of diameter $\leq 1 / 4$. We carry on in this way, so that $U_{\sigma \mid n}$ has diameter $\leq 1 / 2^{n}$.

It then remains to prove the following assertion:

$$
G(\Gamma)=\bigcup_{\sigma} \prod_{n=1}^{\infty}\left(A_{\sigma \mid n}{ }^{\times B_{\sigma \mid n}}\right),
$$

where for each finite sequence $\sigma \mid n$ of integers,

$$
A_{\sigma \mid n}=\Omega^{-}\left(U_{\sigma \mid n}\right) \text {, and } B_{\sigma \mid n}=\varphi\left(U_{\sigma \mid n}\right)^{-} \text {. }
$$

Now if $(t, x) \in G(\Gamma)$, there exists a point $y$ in $P$ such that $(t, y) \in G(\Omega)$ and $x=\varphi(y)$.

There also exists a sequence $\sigma$ of positive integers such that $\{y\}=\bigcap_{n=1}^{\infty} U_{\sigma \mid n}$

Therefore $\Omega(t)$ meets $U_{\sigma \mid n}$ for each $n$, and $x \in \varphi\left(U_{\sigma \mid n}\right)$ for each $t$

Therefore $(t, x) \in A_{\sigma \mid n} \times B_{\sigma \mid n}$ for all $n$.

Conversely, suppose that there exists a sequence $\sigma$ such that $(t, x) \in A_{\sigma \mid n} \times B_{\sigma \mid n}$ for all $n$. 
Then $U_{\sigma \mid n}$ meets $\Omega(t)$ for each $n$, in $y_{n}$ say, and since $(P, d)$ is a complete space, $y_{n} \rightarrow y$, say, as $n \rightarrow \infty$. Since $\Omega(t)$ is closed, $y \in \Omega(t)$.

Then $x=\varphi(y)$; for, if not, there exists a closed neighbourhood $V$ of $\varphi(y)$ such that $x \notin V$. Since $\varphi$ is continuous, $\varphi\left(U_{\sigma \mid n}\right) \subset V$ for some $n$, whence $B_{\sigma \mid n} \subset V$, which contradicts our assumption that $x \in B_{\sigma \mid n}$ for all $n$.

Therefore $(t, x) \in G(\varphi \circ \Omega)=G(\Gamma)$, as required.

The converse also holds, under slightly different hypotheses.

THEOREM 6. Let $S$ be a measurable space which admits the Souslin operation, $X$ a space which is the continuous image of a Polish space, and $\Gamma: S \rightarrow X$ a multifunction with Souslin-R graph. Then $\Gamma$ is of Souslin type.

Proof. Suppose that $X=\varphi(P)$, where $P$ is a Polish space, and that

$$
G(\Gamma)=\bigcup_{\sigma} \bigcap_{n=1}^{\infty}\left(A_{\sigma\left|n^{\times B} \sigma\right| n}\right),
$$

where each $A_{\sigma \mid n}$ is measurable and each $B_{\sigma \mid n}$ is closed. Let $N$ be the space of positive integers with the discrete topology and take the Polish space $N^{N}$, the product of $N$ with itself countably many times. Consider now the multifunction $\Omega: S \rightarrow P \times N^{N}$ defined by

$$
G(\Omega)=\bigcup_{\sigma} \bigcap_{n=1}^{\infty}\left[A_{\sigma \mid n} \times\left(B_{\sigma \mid n}^{*} \times C_{\sigma \mid n}\right)\right],
$$

where for each $\sigma \mid n, B_{\sigma \mid n}^{*}=\varphi^{-1}\left(B_{\sigma \mid n}\right)$ and

$$
c_{\sigma \mid n}=\left\{\tau \in N^{N}: \tau_{1}=\sigma_{1}, \ldots, \tau_{n}=\sigma_{n}\right\} \text {. }
$$

The sets $C_{o \mid n}$ are clearly closed. $\Omega$ is a measurable multifunction, by the Corollary 2 to Lemma 1 .

Moreover, it is closed-valued; suppose that $\Omega(t) \neq \varnothing$ and that 


$$
(x, \kappa) \notin \bigcap_{n=1}^{\infty}\left(B_{\sigma \mid n}^{*} \times C_{\sigma \mid n}\right)=\bigcap_{n=1}^{\infty} B_{\sigma \mid n}^{*} \times\{\sigma\}
$$

There are two cases: if $\prod_{n=1}^{\infty} A_{K \mid n}=\emptyset$, then the neighbourhood $X \times\{\kappa\}$ of $(x, \kappa)$ does not meet $\Omega(t)$. On the other hand, if $\bigcap_{n=1}^{\infty} A_{\kappa \mid n} \neq \emptyset$, then $x \notin \bigcap_{n=1}^{\infty} B_{K \mid n}^{*}$, and since the latter is a closed set, there exists a neighbourhood $U$ of $x$ which does not intersect it. Then the neighbourhood $U \times\{k\}$ of $(x, k)$ does not meet $\Omega(t)$.

Finally, let $\psi$ be the mapping $\varphi \circ \pi_{1}$, where $\pi_{1}$ is the natural projection $P \times N^{N} \rightarrow P$. Then for each $t, \Gamma(t)=\psi(\Omega(t))$, and so $\Gamma$ is of Souslin type.

This last step needs proof: if $(t, x) \in G(\Gamma)$, then for some $\sigma$, $(t, x) \in A_{\sigma \mid n} \times B_{\sigma \mid n}$ for all $n$. Suppose also that $x=\varphi(y)$. Then $y \in B_{\sigma \mid n}^{*}$ for all $n$, and $(t, y, \sigma) \in G(\Omega)$.

COROLLARY 1. If $S$ is a measurable space which admits the sousin operation, and $X$ a Hausdorff space, then the class of miltifunctions of Souslin type from $S$ into $X$ is closed with respect to the Souslin operation.

Proof. Let $\left(\Gamma_{\sigma \mid n}\right)$ be a countable family of multifunctions of Souslin type from $S$ into $X$. Let the corresponding Polish spaces and continuous mappings be $P_{\sigma \mid n}$ and $\varphi_{\sigma \mid n}$. Then without any loss of generality we may replace $X$ by the space $\varphi(P)$ where $P$ is the sum (or disjoint union) of the spaces $\left(P_{\sigma \mid n}\right)$, and $\varphi: P \rightarrow X$ is a continuous mapping which coincides with $\varphi_{\sigma \mid n}$ on $P_{\sigma \mid n}$. Let

$$
\Gamma(t)=\bigcup_{\sigma}^{U} \prod_{n=1}^{\infty} \Gamma_{\sigma \mid n}(t) \text { for each } t
$$

Now each $G\left(\Gamma_{\sigma \mid n}\right)$ is Souslin-R in $S \times \varphi(P)$, and hence, by $\$ 19$ of [6], $G(\Gamma)$ is Souslin-R. Hence $\Gamma$ is of Souslin type, by Theorem 6 .

COROLLARY 2. If $S$ is a measurable space which admits the Souslin 
operation, and $X$ a Hausdorff space, then the class of multifionctions of Souslin type from $S$ into $X$ is closed with respect to the formation of countable intersections.

\section{The existence of measurable selectors}

We first prove a lenma which will enable us to deduce the measurable selection theorem of [14].

LEMMA 3. Let $(S, M)$ be a measurable space which adrits the Souslin operation, $X$ a space which is the continuous image of a Polish space, and $\Gamma: S \rightarrow X$ a multifunction such that $G(\Gamma)$ belongs to the o-algebra $M \otimes B_{X}$. Then $\Gamma$ is of Souslin type.

Proof. We prove this first of all in the case where $X$ is a Polish space. It is sufficient to prove that each set in $M \otimes B_{X}$ is Souslin-R. Let $A$ be the class of sets $A$ in $S \times X$ such that both $A$ and its complement $A^{\prime}$ are Souslin-R. This class is a $\sigma$-algebra, since by [6], \$19, any Souslin class is invariant under the formation of countable unions and intersections. A also contains the sets of $R$, for if $M$ is measurable and $F$ is closed, the complement of $M \times F$ is

$$
(M \times P)^{\prime}=\left(M^{\prime} \times X\right) \cup\left(S \times F^{\prime}\right),
$$

which is clearly Souslin-R.

The sets $M \times F$ generate the $\sigma$-algebra $M \otimes B_{X}$ since the $\sigma$-algebra generated by the sets $\{M \times F: F$ closed in $X\}$ for a fixed set $M$ in $S$ contains all the sets $M \times B$ where $B$ is a Borel set in $X$.

Hence $G(\Gamma)$ is Souslin-R, and so $\Gamma$ is of Souslin type, by Theorem 6.

Suppose now that $X=\varphi(P)$, where $P$ is a Polish space and $\varphi$ a contimuous mapping. Consider the mapping

$$
\psi: S \times P \rightarrow S \times X
$$

defined by $\psi(t, p)=(t, \varphi(p))$.

The inverse image of any "rectangle" $A \times B$ in $S \times X$ is the rectangle $\dot{A} \times \varphi^{-1}(B)$ in $S \times P$, and hence the inverse under $\psi$ of any 
set in $M \otimes B_{X}$ must belong to $M \otimes B_{P}$.

Thus, if $G(\Gamma) \in M \otimes B_{X}$, the multifunction $\varphi^{-1} \circ \Gamma$ is of Souslin type, and hence $\Gamma$ is of Souslin type, by Lemma 2.

We now turn to the problem of the existence of measurable selectors. If $\Gamma: S \rightarrow X$ is a multifunction with non-empty values, a selector for $\Gamma$ is a function $\gamma: S+X$ such that $\gamma(t) \in \Gamma(t)$ for every $t$ in $S$. Also $\gamma$ is measurable if $\gamma^{-1}(F)$ is measurable for every closed set $F$ in $X$.

Now let $S$ be any measurable space and $X$ a Polish space; if $\Gamma$ is a closed-valued multifunction from $S$ into $X$, and its values are all non-empty, then it follows from the main theorem of [9] and from the proof of Théorème 5.4 of [3] that there exists a countable collection $\left\{r_{n}: n=1,2, \ldots\right\}$ of measurable selectors of $\Gamma$ such that for each $t \in S$, the set $\left\{\gamma_{n}(t): n=1,2, \ldots\right\}$ is dense in $\Gamma(t)$. Hence we have:

THEOREM 7. If $S$ is a measurable space, $X$ a topological space and $\Gamma: S \rightarrow X$ a multifunction of Souslin type with non-empty values, then there exists a sequence $\left(\gamma_{n}\right)$ of measurable selectors of $\Gamma$ such that for each $t$ the set $\left\{\gamma_{n}(t): n=1,2, \ldots\right\}$ is dense in $\Gamma(t)$.

Proof. There exist a Polish space $P$, a continuous mapping $\varphi: P \rightarrow X$, and a measurable closed-valued multifunction $\Omega: S \rightarrow P$ such that $\Gamma(t)=\varphi(\Omega(t))$ for each $t$.

Since $\Gamma(t) \neq \varnothing$ for all $t, \Omega(t) \neq \emptyset$ for all $t$. Therefore $\Omega$ has a countable dense collection $\left(\omega_{n}\right)$ of messurable selectors. The theorem follows on putting $r_{n}=\varphi \circ \omega_{n}, n=1,2, \ldots$.

In particular, from Lemma 3 and Theorem 7, we deduce Théorème 1 of [14] in the following form:

COROLLARY. If $S$ is a measurable space which admits the Sousin operation, $X$ the continuous image of a Polish space, and $I: S \rightarrow X$ a multifunction with non-empty values such that $G(\Gamma) \in M \otimes B_{X}$, then there 
exists a sequence $\left(\gamma_{n}\right)$ of measurable selectors of $\Gamma$ such that for each $t$ the set $\left\{\gamma_{n}(t): n=1,2, \ldots\right\}$ is dense in $\Gamma(t)$.

Finslly we give an application of this theory. Implicit function theorems and "lifting theorems" (see, for example, [10]) have been found useful for the abstract theory of control. We give two examples of a type of implicit function theorem known in the literature as Filippov's Lemma, from its initial appearance in [5].

THEOREM 8. Let $(S, M)$ be a measurable space which admits the Souslin operation, $X$ a Hausdorff space, and $Y$ a separable metri able space. Let $f: S \times X+Y$ and $g: S \rightarrow Y$ be finctions measurable with respect to $M \otimes B_{X}$ and $M$ respectively. Suppose that $\Gamma: S+X$ is a multifunction of Souslin type such that, for each $t, g(t) \in f(t, \Gamma(t))$ Then $\Gamma$ has a measurable selector $\gamma$ such that $g(t)=f(t, \gamma(t))$ for each $t$.

THEOREM 9. Let $S$ be a locally compact Hausdorff space on which is defined a Radon measure $\mu$. Let $X, Y$ be Hausdorff spaces, $f: S \times X \rightarrow Y$ a continuous fronction, $\Gamma: S \rightarrow X$ a multifinction of Souslin type, and $g: S \rightarrow Y$ a $\mu$-measurable function such that, for each $t, g(t) \in f(t, \Gamma(t))$. In addition, suppose that $X$ is regular. Then $\Gamma$ has a measurable selector $\gamma$ such that for each $t$, $f(t, \gamma(t))=g(t)$.

(By $\mu$-measurable we mean that given any compact set $K$ in $S$ and any positive real number $\varepsilon$, there exists a compact set $K_{\varepsilon} \subset K$ such tha $|\mu|\left(K \backslash K_{\varepsilon}\right) \leq \varepsilon$ and $g \mid K_{\varepsilon}$ is continuous.)

The proof in each case consists of showing that the multifunction $\Delta: t \rightarrow\{u \in X: f(t, u)=g(t)\}$ is of Souslin type.

By various ways, such as identifying $S$ and $Y$ or by taking $\Gamma$ to be constant, we may deduce all the implicit function theorems to be found in [11] (Lemma 5, p. 448), [1], [3], [7], [12]. (In the cases where $X$ has to be embedded in its completion, we may restrict $f$ to the graph of $\Gamma$; so Theorems 8 and 9 still hold if $\Gamma$ is a measurable compact-valued multifunction and $X$ is a separable metric space.) 


\section{References}

[1] Robert J. Aumann, "Integrals of set-valued functions", J. Math. Anal. App 2. 12 (1965), 1-12.

[2] Nicolas Bourbaki, Elements of mathematics. General topology, Part 2 (Hermann, Paris; Addison-Wesley, Reading, Massachusetts; Palo Alto; London; Don Mills, Ontario; 1966).

[3] Ch. Castaing, "Sur les multiapplications mesurables", Rev. Frangaise Informat. Recherche Opérationelle 1 (1967), 91-126.

[4] Gerard Debreu, "Integration of correspondences", Proceedings of the fifth Berkeley symposizm on mathematical statistics and probability, Volume II, Part 1, 351-373 (University of California Press, Berkeley and Los Angeles, 1967).

[5] A.F. Filippov, "On certain questions in the theory of optima] control", SIAM J. Control Ser. A 1 (1962-1963), 76-84.

[6] Felix Hausdorff, Set theory (translated by John R. Aumann, et al. Chelsea, New York, 1957).

[7] C.J. Himmelberg and F.S. van Vleck, "Selection and implicit function theorems for multifunctions with Souslin Braph", Bul2. Acad. Polon. Sci. Sér. Sci. math. astronom. phys. 19 (1971), 911-916.

[8] K. Kuratowski, Topology I (New edition, revised and augmented. Translated by J. Jaworowski. Academic Press, New York and London; Panstwowe Wydawnictwo Naukowe, Warsaw; 1966).

[9] K. Kuratowski and C. Ryll-Nardzewski, "A general theorem on selectors", Buzz. Acad. Polon. Sci. Sér. Sci. math. astronom. phys. 13 (1965), 397-403.

[10] E.J. McShane and R.B. Warfield, Jr., "On Filippov's implicit functions lemma", Proc. Amer. Math. Soc. 18 (1967), 41-47.

[11] John von Neumann, "On rings of operators. Reduction theory", Ann. of Math. (2) 50 (1949), 401-485.

[12] A.P. Robertson, "On measurable selections", Proc. Roy. Soc. Edinburgh Ser. A 72 (1972-73), 1-7 (1974). 
[13] C.A. Rogers, Bousdorff measures (Cambridge University Press, London, New York, 1970).

[14] Mlle Marie-France Sainte-Beuve, "Sur la généralisation d'un théorème de section mesurable de von Neumann-Aumann et applications à un théorème de fouctions implicites mesurables et à un théorème de représentation intégrale", C.R. Acad. Sci. Paris, Ser. A 276 (1973), 1297-1300.

Department of Mathematics,

University of Western Australia,

Nedlands,

Western Australia. 